\title{
Scanning Surface Potential Microscopy of Bacteriorhodopsin Mutant D96N
}

\author{
I. Lee, ${ }^{*}$ J. A. Stuart, ** S. Budy, ${ }^{* *}$ J. R. Hillebrecht, ${ }^{* * *}$ R. R. Birge, ${ }^{* * *}$ and Elias Greenbaum**** \\ * Department of Electrical Engineering, University of Tennessee, Knoxville, Tennessee 37996-2100 \\ ** Department of Chemistry, Syracuse University, Syracuse, New York 13244 \\ ***Department of Chemistry, University of Connecticut, Storrs, CT 06269 \\ ****Chemical Science Division, Oak Ridge National Laboratory, Oak Ridge, TN 37831-6194
}

Bacteriorhodopsin (BR) is a component of the purple membrane of the salt marsh bacterium Halobacterium salinarum. Absorption of light by the chromophore cause a femtoseconds shift of negative charge, that initiates a picoseconds all-trans to 13-cis photoisomerization followed by a complex photochemical cycle that involves several sequential intermediate states labeled J, K, L, M, $\mathrm{N}$, and $\mathrm{O}$, before returning to the initial BR state in approximately $10 \mathrm{~ms}$. BR has received much attention for biomolecular electronic device applications [1]. While the native protein can be used in various devices, chemical or mutagenic modification is often required for optimized performance. The mutant $\mathrm{D} 96 \mathrm{~N}$ has a longer M state lifetime due to the replacement of aspartic acid (Asp-96) by asparagine (Asn-96) [2]. The charge motions inside the protein are strongly $\mathrm{pH}$ dependent and a longer lifetime was observed with increasing pH [3]. Previously, we used the surface potential microscopy (SSPM) technique to obtain quantitative values of the light-induced photovoltage at nanometer resolution from single photosynthetic reaction centers that were immobilized on gold [4]. This technique can also be used to study electrostatic potentials at the air-liquid interface [5].

We report here the direct measurement of photoinduced surface potential differences of wild-type (WT) and mutant D96N bacteriorhodopsin membranes at $\mathrm{pH} 7$ and 10.5. AFM and SSPM were used to measure the bacteriorhodopsin membrane crystal with the extracellular side facing up. We obtained AFM and SSPM images of WT and mutant D96N in which the light-dark transition occurred in the mid-scan of a single BR membrane. The photoinduced surface potential of D96N is $63 \mathrm{mV}$ (see Fig. 1) at $\mathrm{pH} 10.5$ and is $48 \mathrm{mV}$ at $\mathrm{pH}$ 7. The photoinduced surface potential of WT is 37 $\mathrm{mV}$ at $\mathrm{pH} 10.5$ and $\sim 0$ at $\mathrm{pH} 7$. The results indicated that the surface potentials were generated by photofomation of surface charges on extracellular side of the membrane. Higher surface potential correlated with a longer lifetime of the charges. For theoretical and mathematical modeling, electrostatic contour maps were generated using the $2.0 \AA$ crystal structures of the BR variant, $\mathrm{D} 96 \mathrm{~N}$, in both the resting $(1 \mathrm{C} 8 \mathrm{R})$ and the late $\mathrm{M}$ state $(1 \mathrm{C} 8 \mathrm{~S})$ of the photocycle [6]. The extracellular surface becomes significantly more positive in the late $\mathrm{M}$ state relative to the bR-like resting state of D96N. The shift in charge is equivalent to a dipole moment change of $31.5 \mathrm{D}$ (see Fig. 2).

\section{References}

[1] R. R. Birge et al., J. Phys. Chem. B 103 (1999) 10746.

[2] H. J. Butt et al., EMBO 8 (1989) 1657.

[3] K. Ludmann et al., Biophys. J. 75 (1998) 3120.

[4] I. Lee et al., J. Phys. Chem. B 104 (2000) 2439.

[5] I. Lee et al., J. Phys. Chem. B 107 (2003) 14225.

[6] H. Luecke et al., Science 286 (1999) 255. 


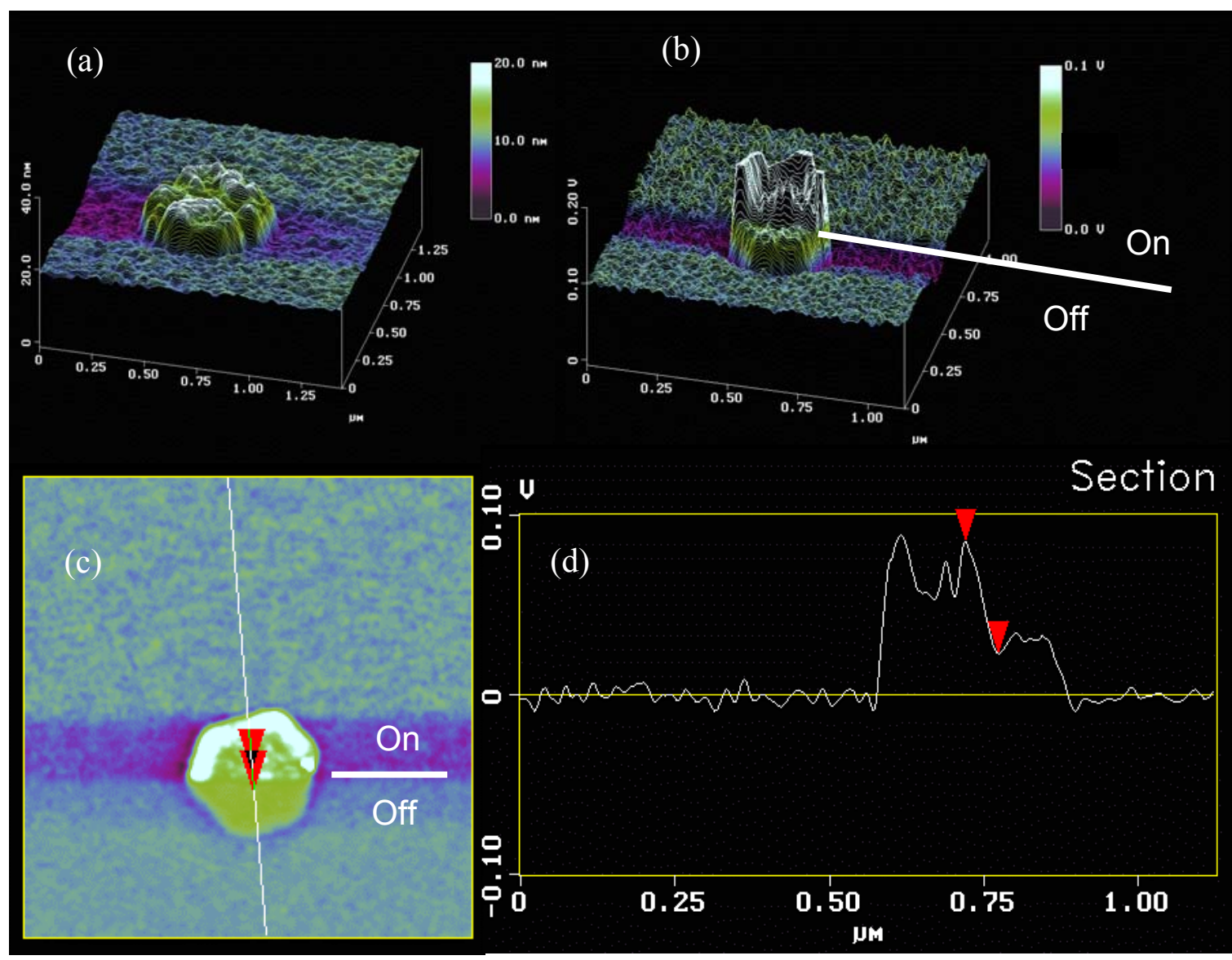

Fig. 1. BR D96N at pH 10.5, (a) was the AFM topographic image, (b) was the corresponding SSPM surface potential image, (d) was the surface potential at the cross section indicated in (c) and the vertical distance between the two pointers was $63 \mathrm{mV}$.

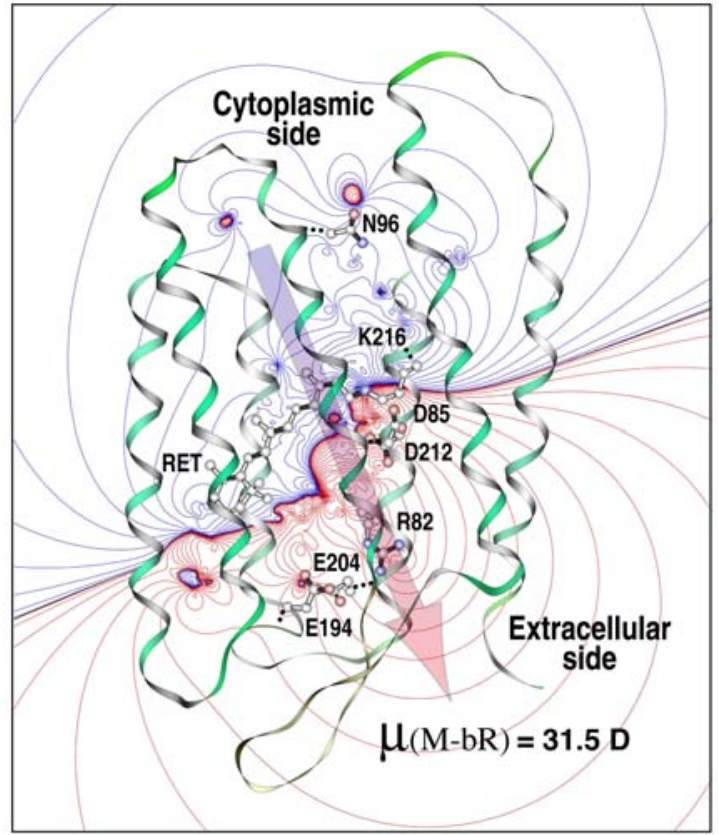

Fig. 2. Electrostatic contours map of the mutant D96N. 